IRA-International Journal of Management \& Social Sciences

ISSN 2455-2267; Vol.05, Issue 01 (2016)

Pg. no. 98-103

Institute of Research Advances

http://research-advances.org/index.php/RAJMSS

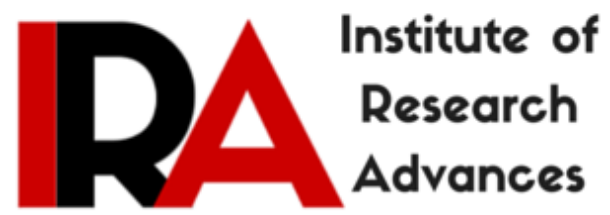

\title{
Quality of life of Persons with Diabetes Mellitus
}

\author{
${ }^{1}$ Esther Oommen \\ MSW, Department of Social Work, Christ University, \\ Bengaluru, Karnataka, India. \\ ${ }^{2}$ Dr. Mathew CP \\ Associate Professor, Department of Social Work, \\ Christ University, Bengaluru, Karnataka, India.
}

Type of Review: Peer Reviewed.

DOI: http://dx.doi.org/10.21013/jmss.v5.n1.p11

\section{How to cite this paper:}

Oommen, Esther, \& CP, Mathew (2016). Quality of life of Persons with Diabetes Mellitus. IRA-International Journal of Management \& Social Sciences (ISSN 2455-2267), 5(1), 98-103. doi:http://dx.doi.org/10.21013/jmss.v5.n1.p11

(C) Institute of Research Advances

\section{(cc) EY-NO}

This work is licensed under a Creative Commons Attribution-Non Commercial 4.0 International License subject to proper citation to the publication source of the work.

Disclaimer: The scholarly papers as reviewed and published by the Institute of Research Advances (IRA) are the views and opinions of their respective authors and are not the views or opinions of the IRA. The IRA disclaims of any harm or loss caused due to the published content to any party. 


\begin{abstract}
Quality of life is considered as the extent to which a person appreciates the essential potential outcomes of his/her life. Potential outcomes result from the open doors and confinements every individual has in his/her life and mirror the cooperation of individual and ecological components. The main aim of this study was to understand the quality of life among the people with diabetes mellitus. The sampling technique used for this study was purposive sampling. WHOQOL-BREF questionnaire was used to assess the quality of life. It was assessed through four domains like physical, psychological, social and environment. Through results it was found that high percentage of study population had low quality of life in all four domains. The researcher also assessed the management of the illness in the people diagnosed with the diabetes mellitus. A self-structured questionnaire was used for this purpose. The results showed that the study population manage their illness through various measures like diet restrictions, follow ups with the doctor, maintained optimal blood sugar, treatment adherence and involve in exercise.
\end{abstract}

Keywords: Quality of Life, Diabetes Mellitus, Management of Diabetes Mellitus

\title{
Introduction
}

\section{Quality of life}

In the quality of life, the being space incorporates the essential parts of "who one is" and has three sub-areas. Physical being incorporates parts of physical wellbeing, individual cleanliness, sustenance, exercise, prepping, garments, and physical appearance. Mental being incorporates the individual's mental wellbeing and alteration, perceptions, emotions, and assessments concerning the self, and poise. Profound being reflects individual qualities, individual guidelines of behavior, and otherworldly convictions which might be connected with sorted out religions. Having a place incorporates the individual's fit with his/her surroundings furthermore have three sub-spaces. Physical belonging is characterized as the associations the individual has with his/her physical surroundings, for example, home, work environment, neighborhood, school and group. Social Belonging incorporates joins with social situations and incorporates the feeling of acknowledgment by personal others, family, companions, colleagues, and neighborhood and group (Quality of Life Research Unit, University of Toronto, n.d.).

\section{Diabetes Mellitus}

Diabetes mellitus is a long term illness that disturbs the body's capacity to consume the vitality found in the intake of food. Diabetes is found in three types: type 1 diabetes, type 2 diabetes, and gestational diabetes. Usually our body disrupts the carbohydrates and sugars into glucose. It energizes the cells. For this process to happen it needs a hormone in our blood which is insulin. To get energy, insulin is needed to convert glucose so that the person can regain his stamina. Either it is unable to generate insulin or the insulin which is produced is not consumed. The glucose in the body is not used consequently hence forms inside the body. Hence this can destruct the various organs in the body (Diabetes health Center, 2016).

The symptoms of diabetes mellitus are: hunger and fatigue, recurrent urination and dehydration, dry mouth and itchy skin, blurred vision, slow healing sores, nausea vomiting (Diabetes health Center, 2016). Type I diabetes is caused by many reasons like genetic, Pancreatic disease results in incapacitating the insulin production. The other type of diabetes is caused by the following reasons: Weight or being fat. A Study demonstrates it as one of the risk factor for this illness 
(Diabetes health Center, 2016). The treatment for diabetes mellitus is given by the insulin pump is intended to convey a ceaseless measure of insulin, 24 hours a day as indicated by a modified arrangement special to every pump wearer. The client can change the measure of insulin conveyed. Other treatments envelop an assortment of controls that incorporate everything from eating regimen and activity to mental molding and way of life changes. Samples of option medications incorporate needle therapy, guided symbolism, chiropractic medicines, yoga, entrancing, biofeedback, fragrance based treatment, unwinding works out, home grown cures, and numerous others (Diabetes health Center, 2016)

\section{Objectives}

The study was focussed on two objectives listed below:

1. To assess the quality of life of the persons with Diabetes Mellitus

2. To assess diabetes mellitus management in the people.

\section{Methodology}

The sampling technique used for this study was purposive sampling. 60 patients with Diabetes Mellitus were interviewed for the purpose of the study from Bengaluru City. WHOQOL- BREF questionnaire was used to assess the quality of life. It was assessed through four domains like physical, psychological, social and environment. Through results it was found that high percentage of study population had low quality of life in all four domains. The researcher also assessed the management of the illness in the people diagnosed with the diabetes mellitus. A self -structured questionnaire was used for this purpose. It had questions regarding the doctor's appointments, whether they are able to maintain the optimal blood sugar level, treatment adherence, involvement in physical exercise etc.

\section{Results and Discussion}

The present study is focussed on patient's quality of life that is diagnosed with Diabetes mellitus. It also looks into the management of this illness in the people. Quality of life in this study is studied based on four different domains in an individual's life. There are major aspects that are studied. It is on different areas of life that affects the patient diagnosed with diabetes mellitus. The illness has adverse effects on the activities of daily living, interpersonal and social skills. It affects the patient's emotional stability. The environmental factors also can hamper the patient's well-being. It includes financial stability of the patient. Hence this study was conducted to gain knowledge about the above aspects. The management of the illness is assessed based whether they follow a diet; involvement in physical activity etc. The sample size for the study was 30 .

\section{Domains of Quality of life}

In physical domain, $86.7 \%$ (52) of the patient's quality of life was low and $13.3 \%$ (8) of the population quality of life was high. There were questions that focus on the physical condition of the persons with diabetes mellitus. It includes the activities of daily living, the energy that the people have, to carry out their normal functioning, whether there is any hindrance for them to move around, physical pain and discomfort. It also focuses on the sleep pattern and their capacity to do their work. It is based on the personal satisfaction that they perceive to have in these different aspects. In diabetes mellitus the person gets easily fatigue and their capacity to work reduces because of their health condition. 
In psychological domain, 53.3\% (32) of the study population's quality of life was low and 46.7\% (28) of their quality of life was high. This domain mainly focuses on their emotions and their psychological state. It has questions on whether they are able to accept their bodily appearance, their consciousness about their body image, whether they enjoy their life, the extent to which they feel their life to be meaningful. It also asks on whether they fear the future and their worries about confronting the death, positive feelings and negative feelings like depression, anxiety, and blue mood is also focused on this domain. Their religion beliefs, spirituality are also assessed through this domain. They feel that they have become a burden to the family due to the illness because of the expenses that the family has to bear for them. They feel that they are dependent on others. They have ruminations that they are not able to shoulder the responsibility that is assigned to them.

In social domain, $86.7 \%$ (52) of the study population's quality of life was low and $13.3 \%$ (8) of the sample's quality of life was high. This was assessed through asking respondents about their interpersonal relationship. There were questions that were asked about their satisfaction with their personal relationship, the social support and their sexual life. The social support is apart from the support they receive from their spouses. It can be their friends, colleagues, extended family etc. Usually the personal relationship is strained when any one of the spouse has some form of illness. A diabetic person usually becomes lethargic so they won't be able to go for any social gatherings. This might lead to their spouse to become upset about it. Sometimes they are deprived of social support. This can be because the people might think it as a burden because the person might be in need of finance for the medications. Every human being has certain inborn instincts and they are motivated to satisfy those instincts. Sex is one of the biological instincts. In diabetic patients there is sexual dysfunction.

In environment Domain, $76.7 \%$ (46) of the sample's quality of life was low and $23.3 \%$ (14) of their quality of life was high. This domain is assessed by asking the respondent's questions regarding their safety, financial resources etc. There were questions like, whether they feel safe in their daily life, do they have enough finance to meet the requirements for their treatment, the freedom that they have in their life, their accessibility to the health services and their perception about the quality of the services provided by the health sector for their better recovery. It also focuses on the opportunities that they derive for their leisure activities. The availability of information to them about their daily life is also one of the aspects in this domain.

This study also throws light on the management of diabetes mellitus in people who are diagnosed with this illness. This was studied by using a self-structured questionnaire. It had questions regarding the doctor's appointments, whether they are able to maintain the optimal blood sugar level, treatment adherence, involvement in physical exercise etc.

\section{Management of Diabetes Mellitus}

The study population was asked whether they continue their follow up with the doctor. $20.0 \%$ (12) of them often had their appointments on track, $13.3 \%$ (8) responded sometimes, $40.0 \%$ (24) occasionally and $26.7 \%$ (16) as rarely. The illness forces the people to constantly consult their doctor to know about the health condition. Some patient's due to their negligence about the illness avoid the follow ups.

The people with diabetics are asked to follow certain medications like insulin injection so that they can maintain their blood sugar level. The increase in blood sugar is because of lack of insulin so they have to follow medications. As per the study 56.7\% (34) of them responded that they 
follow the routine medications, 20\% (12) of their response was sometimes and 23\% (14) occasionally. Some patients lack the knowledge about the medicines that has to be taken immediately after their meals. There are financial constraints that are contributing to the lack of treatment adherence amongst the people.

Physical activity is necessary to maintain their sugar level. So the respondents were asked that whether they engage in physical activity.36.7\% (22) of them responded that often they involve in doing exercise, $33.3 \%$ (20) of the response was sometimes, $5 \%$ (10) of them occasionally and $13.3 \%$ (8) never engage in physical activity.

There are certain dietary restrictions that the doctor asks the diabetic patients to follow. They are asked to consume more fibrous food like brown rice, sweet potato, whole wheat pasta and intake of carbohydrates should be reduced. So the respondents in this study were asked whether they follow a particular diet. $10.0 \%$ (6) of response was often, $36.7 \%$ (22) of the samples followed the diet sometimes, $23.3 \%$ (14) of them occasionally $20.0 \%$ (12) rarely and $10.0 \%$ (6) were never conscious about their diet. The dietary restrictions are not followed since they are not able to resist their temptations. The food they had earlier consumed might be a craving for them to consume again even though they have the illness. Some are not willing to change their lifestyle which is causing a hindrance to restrict their diet.

In illness like diabetes mellitus, if health is neglected there can be complicated illness associated like diabetic retinopathy, which affects the eyes. So regular follow ups with the doctor is necessary. The participants were asked whether they feel that they should visit the medical practioner.23.3\% (14) of them responded yes, no response was given by $33.3 \%$ (20) of the population and $43.3 \%$ (26) of the sample felt the need to visit the medical practitioner. Some people have a different attitude towards this medical illness. The attitude is that the wealth is getting wasted but health is not improving so they have the opinion that there is no requirement that they should consult the medical services.

In this study the patients were asked about whether they are able to maintain the required blood sugar level.16.7\% (10) of the study population responded that they often try to maintain, $40 \%$ (24) of them sometimes $20 \%$ (12) of them occasionally and $23.3 \%$ (14) rarely. This can be because of the negligence that they possess about the illness. The main reason for diabetes mellitus is increased blood sugar level. Hence the patients should strive to control and attain the required sugar level

\section{Conclusion}

The researcher conducted a study on the quality of life of patients with diabetes mellitus. The study revealed that the quality of life is low in many people. In quality of life four domains were assessed. The four domains were physical, psychological, social and environment domain. In all the four domains the quality of life is low. Through this study it was found that people manage their illness through various measures like following a particular diet which would help them to maintain their sugar level. They involve in exercise, follow the medications, they maintain their optimal blood sugar. The people with diabetes mellitus can have a better health if they constantly involve in self-management of the illness. This can be enhanced with the required support and cooperation from the family. Better quality of life can have more longevity in the people with the illness. 


\section{References}

Davies, M. J. (2008). Effectiveness of the diabetes education and self management for ongoing and newly diagnosed (DESMOND) programme for people with newly diagnosed type 2 diabetes: cluster randomized control group. BMJ.

Diabetes health Center. (2016). Retrieved from Webmed: http://www.webmd.com/

Huang, E. S. (2007). Patient Perceptions of Quality of Life With Diabetes-Related Complications and Treatments. Diabetes journal.

Hwang, S. (2000). Barriers to appropriate diabetes management among homeless people in Toronto. CMAJ.

Janssen Quality-of-life Studies. (n.d.). Retrieved from Notes on quality of life: http://www.gdrc.org/uem/qol-define.html

Kiadaliri, A. A. (2013). Quality of life in people with diabetes: a systematic review of studies in Iran. Journal of diabetes and metabolic disorders.

Klein, H. A. (2013). Diabetes Self-Management Education: Miles to Go. Hindawi.

Lindsay, G., \& McDowell, J. R. (2011). Quality of Life in People with Type 2 Diabetes in Relation to Deprivation, Gender, and Age in a New Community-Based Model of Care. Nursing Research and Practice, 8.

Norris, S. (2002). Self management Education for Adults with Type 2 Diabetes. Diabetes Care.

Nyanzi, R. (2014). Diabetes and Quality of Life: A Ugandian Perspective. Journal of Diabetes Research.

Olivia, J. (2012). Health- related quality of life in diabetic people with different vascular risk. BMC Public Health.

Papadopoulos, A. A. (2007). Predictors of health-related quality of life in type II diabetic patients in Greece. Biomed Central.

Spasic, A. (2014). Quality of Life in Type 2 Diabetic Patients. Scientific Journal of the Faculty of Medicine, 193-200.

WHOQOL-BREF Introduction, Administration, Scoring and Generic Version of the Assessment. (n.d.). Retrieved February Tuesday, 2015, from WHO Mental Health Media: http://www.who.int/mental_health/media/en/76.pdf 